\title{
ECONOMÍA PARA NO MORIR. Un análisis de las necesidades mínimas vitales y su metabolismo con el planeta tierra
}

\author{
Carlos Humberto Salas Cardona y Liceth Cristina Ramírez Hurtado. Universidad del Cauca, \\ Popayán, Colombia \\ Recepción: 7 de septiembre de 2015 | Revisión: 8 de septiembre de 2015 | Aceptación/Publicación: 18 de septiembre de 2015 \\ Correspondencia: salasagiio@gmail.com | crisramirez2130@gmail.com \\ Citar: Salas Cardona, CH. y Ramírez Hurtado, LC. (2015). ECONOMÍA PARA NO MORIR. Un análisis de las necesidades mínimas vitales y \\ su metabolismo con el planeta tierra. ReiDoCrea, 4, 245-259. http://hdl.handle.net/10481/37451
}

\begin{abstract}
Resumen: Economía para no morir es una propuesta teórica que parte del análisis y reconocimiento de las necesidades mínimas vitales (NMV) del ser humano, aquellas que le garantizan su condición de vida y poseen como característica principal la transmodalidad. Tal análisis se desarrolla sobre las bases del pensamiento complejo y por tanto bajo un enfoque holístico que redime la subjetividad con que han sido tratadas por estudiosos del tema desde distintas posturas teóricas; así, con aportes de la Filosofía, la Antropología, la Biología, la Sociología, la Medicina y por supuesto la Economía, sentamos las bases para la identificación y caracterización de aquellas NMV como un subconjunto base de las necesidades humanas. Una vez expuestas, actúan como variables guía en la construcción metodológica de una herramienta capaz de identificar su insatisfacción para cualquier grupo humano, el Índice mínimo vital (IMV). La materialización de ésta propuesta pone la vida antes que los demás derechos fundamentales, visibilizando la interacción metabólica del ser humano con el planeta tierra en la búsqueda constante de satisfacción de aquellas NMV.
\end{abstract}

Palabras claves: Necesidades básicas | Desarrollo económico y social

ECONOMY NOT TO DIE. An analysis of the vital minimum needs and their metabolism with earth planet

\begin{abstract}
Economy not to die is a theoretical design, which starts from the analysis and recognition of the vital minimum, needs (VMN) of human beings, those that guarantee its life condition and have as a main feature the trans-modality. Such analysis is developed on the basis of complex thought and therefore under a holistic approach that redeems the subjectivity, which has been treated by scholars from different theoretical postures; thus, with Philosophy, Anthropology, Biology, Sociology, Medicine and Economy contributions, basis are established for the identification and characterization of those VMN as a base of human needs subset. Once exposed, these proposals act as trail variables in the building of a methodological tool able to identify its dissatisfaction to any human group, the vital minimum index (VMI). This proposal materialization gives life before the other fundamental rights, making visible the human metabolic interaction with the earth planet in the constant search of satisfaction of those VMN.
\end{abstract}

Keywords: Basics needs | Economic and Social Development

\section{Introducción}

Hace aproximadamente 195.000 años la especie humana existe en el planeta, desde entonces, compartimos las características anatómicas y necesidades fisiológicas que garantizan nuestra existencia, es decir, cualquier humano expuesto al hambre, la falta de abrigo, poco descanso, carencia de oxígeno, abandono o sed extrema inevitablemente morirá. Tales condiciones básicas para la permanencia en la vida, han sido vulneradas a lo largo de la historia de la humanidad, día a día las padecen millones de personas alrededor del mundo y son la causa principal de un sinnúmero de muertes. Ellas, no solo responden a estados físicos o impulsos biológicos del ser humano, en su mayoría develan una deconstrucción social resultado de diversas actividades socioeconómicas en Colombia y el mundo; justamente, el folleto informativo número 34 de la Organización de las Naciones Unidas para la Agricultura y la Alimentación (FAO), mostró que más de 1.000 millones de personas sufren desnutrición, debido a ello, aproximadamente seis millones de niños y niñas mueren todos los años a causa de enfermedades que se pueden prevenir; a diferencia de lo que pueda pensarse solo el $10 \%$ de esas muertes son resultado de conflictos armados, catástrofes naturales 0 condiciones climáticas 
excepcionales, e increíblemente, el $90 \%$ restante es víctima de la falta de acceso a una alimentación adecuada en forma crónica y en el largo plazo a raíz de políticas de gobierno que generan crecimiento económico empobrecedor (FAO).

Por lo anterior, apelando al derecho fundamental a la vida de todo ser humano, se examinan aquellas condiciones de vulnerabilidad humana al conectarlas a necesidades de urgente satisfacción, visibilizando un subconjunto garante de vida denominado Necesidades Mínimas Vitales (NMV), de manera que, la carencia de al menos una de ellas es un atentado directo a la vida y al derecho fundamental que la ampara. Economía para no morir, un análisis de las necesidades mínimas vitales y su metabolismo con el planeta tierra es una apuesta teórica que permite abordar tal problemática social desde diferentes posturas analíticas de las condiciones fundamentales de vida.

Tal vinculación ilustra dos esquemas; el primero, identifica las NMV y la interacción del ser humano con el planeta tierra; y el segundo, visibiliza interrelaciones, que permiten la comprensión de una cosmovisión sobre la incidencia de la actividad económica en el entorno natural del ser humano; este análisis pone la vida como prioridad de la Economía para no morir e induce que en el centro necesariamente esté el planeta tierra, procurando una condición primordial de los pactos sociales, la vida misma.

\section{Las necesidades humanas y las necesidades mínimas vitales}

Es fácil caer en la subjetividad al analizar el ser humano y su proceso en la historia del planeta tierra, y de entrada se establece el vínculo porque la relación de existencia es inequívocamente dependiente, pues sin el planeta tierra la especie humana no existiría tal como la conocemos, y recíprocamente sin la humanidad, la tierra sería un lugar completamente diferente, escapando a cualquier razonamiento propio de los seres humanos o simplemente no existiría. Ésta subjetividad es el resultado de las profundas separaciones entre ciencias como la filosofía, la economía, la antropología, la biología, el derecho y la medicina, que bajo la rigidez de sus análisis, alejan las condiciones de vida de una visión holística del ser humano para considerarlas como objetos de estudio desligados entre sí. "Sólo un enfoque transdisciplinario nos permite comprender, por ejemplo, de qué manera la política, la economía y la salud han convergido hacia una encrucijada. [...] la mala salud es el resultado de la mala política y de la mala economía" (Max Neef, 1993, p. 39).

El ser humano como punto de partida de todo conocimiento sobre sí mismo y sobre el mundo, es el puente entre las ciencias sociales, humanas y naturales. Por ello, para la evaluación de las necesidades humanas y en este caso particular, las $N M V$, no basta con un análisis en el cual las ciencias identifiquen desagregadamente condiciones de conservación de la vida; es importante comprender el ser humano en su condición orgánica elemental evaluado como un todo. Esto visibiliza las particularidades anatómicas que comparte la especie humana desde hace aproximadamente 195.000 años, es decir, que guardando proporciones evolutivas y de adaptabilidad, todos cuentan con las mismas necesidades fundamentales, lo que varía es la cantidad de satisfactores que debieron consumirse en cada momento histórico para evitar la muerte de personas en el mundo por la privación de alguna o varias NMV.

En este sentido, el ser humano y su bienestar son la principal preocupación antes de llevar a cabo toda actividad socioeconómica y por tanto, el camino trazado parte de la 
ciencia médica que identifica las $N M V$ como condicionantes de la vida al vincular la insatisfacción de ellas a situaciones de muerte, hablamos de hipotermia (Abrigo), asfixia (Oxigenación), inanición (Alimentación), deshidratación (Hidratación), abandono (Amparo) e insomnio fatal (Descanso); cada una de las cuales responde a una necesidad mínima vital que legitima su análisis e identificación. A partir de esto, su categorización en la teoría económica, se construye con base en los planteamientos de Marx (Antropología Filosófica), Doyal - Gough (Sociología Humanista) y Max Neef (Economía) quienes reconocieron la existencia de necesidades de satisfacción primaria como naturales, básicas y de subsistencia respectivamente; dentro de las cuales aunque no explícitamente, se encuentran las NMV.

La teoría de las necesidades humanas en Marx, hace visible una reflexión sobre la esencia humana, las capacidades y las necesidades organizándolas en categorías analíticas; en ese sentido, la esencia humana es definida a través de sus necesidades, capacidades, y las personas son influenciadas por satisfactores, en palabras de Marx, por los objetos de las necesidades. Esta construcción marxista del ser humano reconoce la existencia de necesidades naturales y otras que están socialmente determinadas, sinónimo de las primeras son las necesidades físicas, y de las segundas agrega, son necesidades que requieren una construcción más humana. En esta medida, aquellas necesidades naturales se manifiestan dirigidas a objetos y están dadas por la constitución biológica de los humanos como animales, pero tras el reconocimiento que los límites naturales se dirigen y consagran en la actividad vital: "[...] Físicamente el hombre vive sólo de estos productos naturales, aparezcan en forma de alimentación, calefacción, vestido, vivienda, etc. [...] el hombre vive de la naturaleza quiere decir que la naturaleza es su cuerpo, con el cual ha de mantenerse en proceso continuo para no morir" (Marx, 1844/1968, p. 112).

Lo anterior no solo concede una categoría diferente al comportamiento de las necesidades que son del orden biológico sino que reconoce las $N M V$, ratificando la existencia de necesidades que bajo formas de satisfacción diferentes, están presentes tanto en los hombres como en los animales, aquellas de tipo natural, determinantes para la garantía de la vida. Y aunque en su enfoque teórico Marx es explícito en afirmar que la sociedad subordina los sentidos humanos a necesidades prácticas burdas que acercan el ser humano a un nivel bestial, también es reiterativo en que las necesidades naturales son las primeras en la escala de satisfacción; el ser humano se identifica como selectivo, pues ante la insatisfacción de al menos una necesidad natural bloquea la satisfacción de todas las demás necesidades humanas.

Un análisis sociológico humanista realza individuos que nacen en medios sociales, modelan su imagen, gustos, costumbres, comportamiento físico y formación intelectual pero que al vivir con lo mínimo, requieren exactamente lo mismo para garantizar su subsistencia. Bajo ésta perspectiva, los planteamientos de Doyal y Gough (1994) exhiben la existencia de necesidades básicas y otras intermedias; las primeras, cubren las condiciones previas para asegurar la participación social y la persecución de fines propios, hablamos de: salud física y autonomía personal, ya que, "Para desempeñarse adecuadamente en sus vidas diarias - no importa a qué se dediquen y en qué contexto cultural- la gente no sólo necesita sobrevivir, necesita también poseer una módica salud básica" (Doyal y Gough, 1994, p.59). 
Cada individuo es único, cuenta con deseos y preferencias personales, sin embargo, las necesidades vitales que nos aquejan son las mismas, en situaciones como la falta de comida, el abrigo para sobrevivir o el aire para respirar no existe elección, ya que, no podemos decidir lo que es indispensable para vivir. Así, aunque los seres humanos cuentan con características esenciales que los diferencian de otros animales, la más importante de ellas, la forma en que se satisfacen las necesidades (la actividad vital del trabajo), el como especie en constante evolución responde a su entorno en sociedad y es en principio una especie animal que requiere por norma satisfacer las NMV para garantizar su existencia.

En consonancia, el análisis de Max Neef muestra como la satisfacción de las necesidades se realiza en un proceso continuo y renovado de la vida: "[...] entendidas, las necesidades como -carencia y potencia- resulta impropio hablar de necesidades que se "satisfacen" o que se "colman». [...] es más apropiado hablar de vivir y realizar las necesidades, y de vivirlas y realizarlas de manera continua y renovada" (Max Neef, 1993, p. 50). Es decir, estar vivo es una condición humana que se precisa en necesitar y sentir. Sin embargo, la identificación de las $N M V$ devela una tensión con la teoría de Max Neef en su primera necesidad propuesta, la de subsistencia, que igual a las de Protección, Afecto, Entendimiento, Participación, Ocio, Creación, Identidad y Libertad se encuentra sujeta a insatisfacción al concebirse como carencia o falta de algo; esto abre la posibilidad a la muerte del ser humano necesitado, ya que dentro de ella están las NMV que indefectiblemente deben ser satisfechas. Si el ser humano no tiene posibilidad de revivir para satisfacer sus otras necesidades, una vez insatisfecha la necesidad de subsistencia no existe posibilidad de volver atrás.

"Las necesidades humanas fundamentales [...] son las mismas en todas las culturas y en todos los periodos históricos. Lo que cambia, a través del tiempo y de las culturas, es la manera o los medios utilizados para la satisfacción de las necesidades" (Max Neef, 1993, p. 27) y "[...] alimentación y abrigo no deben considerarse como necesidades, sino como satisfactores de la necesidad fundamental de subsistencia" (Max Neef, 1993, p. 41). Estas dos afirmaciones del mismo texto visibilizan otra tensión; si los satisfactores de la necesidad de subsistencia pueden variar "en todas las culturas y en todos los periodos históricos" (Max Neef, 1993, p. 27) en ellas Max Neef pone a consideración que en cualquier otro momento en la historia de la humanidad hubo o habrá otro satisfactor de la subsistencia diferente a la alimentación y el abrigo, es decir, en algún momento el ser humano pudo sobrevivir sin alimentación y abrigo ya que son consideradas satisfactores. Por esta razón, la subsistencia o lo que hemos denominado a lo largo de Economía para no morir como la permanencia en la vida, es más que una necesidad sujeta a insatisfacción, una condición de todos los seres humanos; y la alimentación, abrigo, respiración, amparo, hidratación y descanso más que satisfactores de la vida son un subconjunto de necesidades garantes de esa condición, siempre hablando en términos fisiológicos. Lejos de ir en contra de la teoría de necesidades humanas de Manfred Max Neef buscamos dar un reconocimiento de condición a su necesidad de subsistencia y establecer de forma específica el sub conjunto de necesidades que la garantizan, las NMV.

\section{Necesidades mínimas vítales: Identificación e insatisfacción}

En el mismo instante en que la opinión pública satura los medios nacionales con imágenes dantescas de miles de cadáveres de animales, víctimas de una extenuante 
sequía que azota el departamento del Casanare, Colombia, durante los últimos meses se revelan datos escalofriantes de mortalidad infantil por deshidratación y desnutrición en el departamento de la Guajira, cerca de 295 niños y niñas han perdido la vida en lo corrido del año a causa de enfermedades relacionadas con la falta de alimentación e hidratación, cifra que se constituye en la punta visible de una problemática que le ha costado a ese departamento 2.969 niñas y niños en los ultimos 5 años según las cifras oficiales, y miles de infantes mas según los cabildos Arawaak, que mueren en el anonimato absoluto de las rancherias Wayuu invisibles para nosotros los y las Arijunas (blancos de occidente).

Colombia, país considerado una despensa agrícola, sexto en el mundo en recursos hídricos, una economía en crecimiento y el mas feliz del mundo, al igual que muchos otros enfrenta una cruda realidad: la desnutrición crónica es endémica, y la padecen el $12 \%$ de los niños según datos obtenidos de la Agencia Fides. En la Guajira, los habitantes coexisten con el Cerrejón, reconocida como la mina a cielo abierto más grande del país, la cual gasta 17 millones de litros de agua para riego diario de caminos mientras que un habitante guajiro consume 0.7 litros diarios de agua no tratada (Robledo, 2014). La crisis que sufre este departamento con más de $\$ 6.5$ billones de pesos recibidos por transferencias y regalías en los últimos 12 años (Robledo, 2014), no solo evidencia el despilfarro en manos de dirigentes corruptos que compran almas y conciencias, más importante es la sobre posición de la acumulación, a la vida humana y el ambiente natural. Situación presente alrededor del mundo y frente a la cual, el Casanare y la Guajira son solo la punta del iceberg.

El planeta brinda los recursos necesarios para satisfacer cada una de nuestras necesidades, y la Economía entendida como ciencia que analiza la forma en que los individuos y la sociedad toman decisiones en función de la optimización de aquellos recursos disponibles, debe contribuir a la satisfacción no solo de necesidades individuales sino del colectivo social, una Economía pensada para la vida como la planteada por Hinkelammert y Mora o en el mismo orden de ideas, una Economía para no morir que pondera primero la vida humana y las condiciones que la permiten.

Situaciones como estas en poblaciones de Colombia y el mundo, identifican una problemática en la interdependencia de sus facetas, biológica, médica, social y ambiental. Por un lado, los datos indican la difícil situación política, socioeconómica y de derechos humanos por la que atraviesan las comunidades, y por otro, son el reflejo de malas decisiones políticas y económicas en consecuencias epidemiológicas; la salud y la condición de vida están negativamente relacionadas. Entendido esto, el cuerpo humano y las necesidades que lo mantienen en funcionamiento no se pueden desvincular del análisis económico como si fuese un sistema homeostático independiente, sujeto de análisis clínicos del ciudadano y ciudadana social, esas necesidades fisiológicas son el primer paso para el desarrollo personal; es preciso acudir a la ciencia médica que durante siglos ha estudiado el cuerpo humano y su funcionamiento, para identificar el subconjunto de las Necesidades Mínimas vitales (NMV) de permanencia en la vida como: hidratación, alimentación, abrigo, descanso, respiración y amparo; Estas NMV deben analizarse articuladas a la esencia misma del ser humano indivisible per se, ya que toda y todo ciudadano trasformador del ambiente natural, necesita primero vivir: 


\section{Ilustración 1. Necesidades Mínimas Vitales}

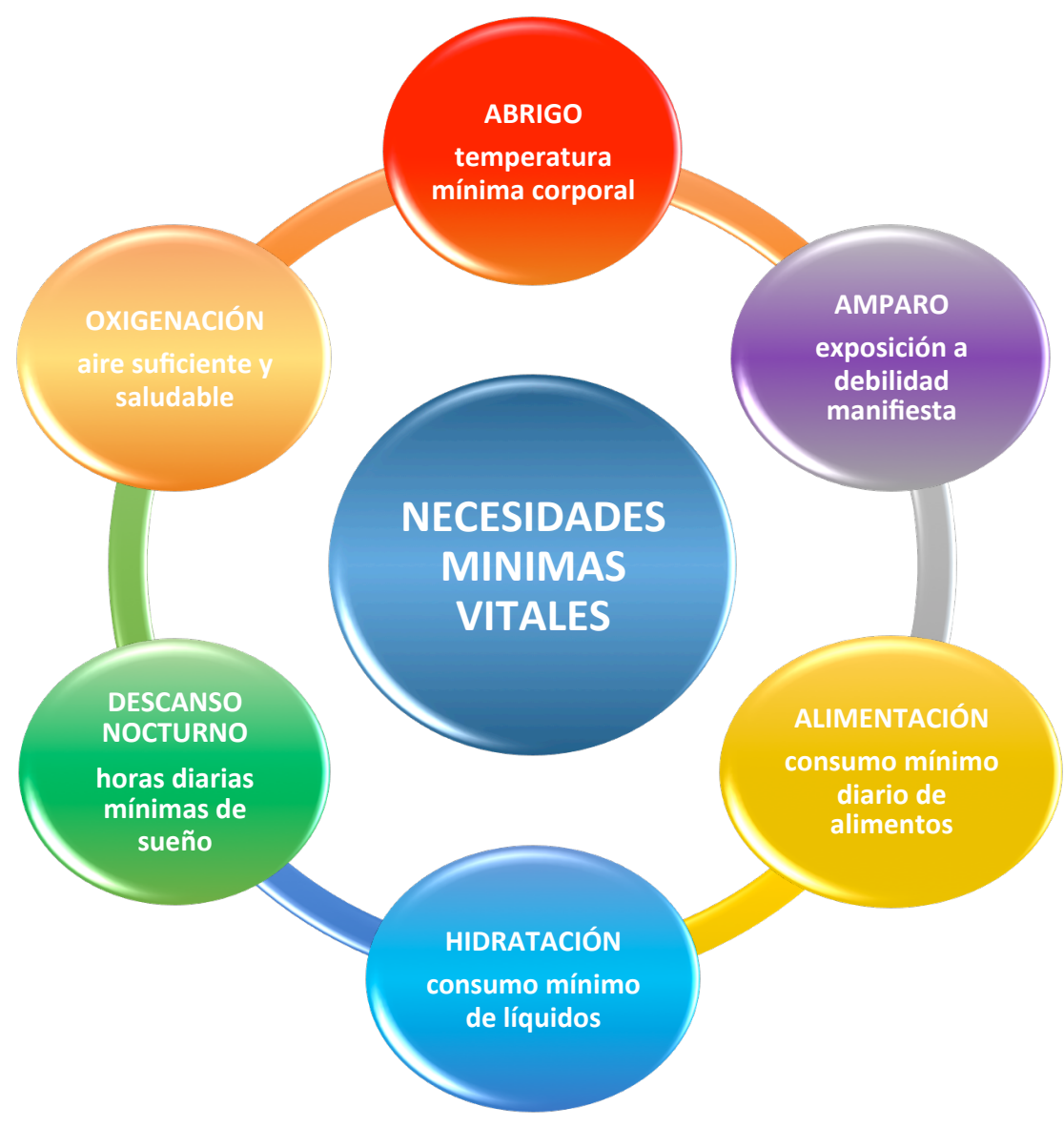

Es preciso aclarar que todas las $N M V$, se postulan como un subconjunto garantes de la vida y por tanto de las necesidades humanas, es decir, son su preámbulo.

\section{Construcción del índice de mínimos vitales (IMV): Herramienta metodológica}

Una vez propuestas las $N M V$, se diseña una herramienta que brinda la posibilidad de identificar la presencia de insatisfacción de dichas necesidades en determinado grupo humano; una metodología que diagnostica la presencia de alguna necesidad mínima vital insatisfecha, y permite utilizar sus resultados en la formulación de políticas públicas encaminadas a la prevención de muertes y de futuros percances.

Tal Índice Mínimo Vital, se define como un indicador capaz de diagnosticar la vulnerabilidad de los integrantes de una comunidad con la vida, producto de la insatisfacción de alguna de ellas. Vinculando elementos cualitativos y cuantitativos con la rigurosidad metodológica de las guías del DANE, y con lineamentos metodológicos de indicadores sociales como el NBI, se estructura para ser utilizado como referente en investigaciones futuras relacionadas con condiciones de vida en diversas regiones de la población colombiana y el mundo. 
Para la construcción metodológica de la herramienta, es esencial reconocer que la característica de todas las $N M V$ es su vínculo con la muerte del ser humano ante la insatisfacción de al menos una de ellas, y por tanto, su importancia radica en la exposición humana a niveles bajos de consumo de bienes que conllevan a la insatisfacción de cualquier NMV.

Al ser las NMV tan diversas, sus satisfactores también son particulares; algunos de ellos son bienes que se pueden transar en el mercado, como el vestido, los refugios, las fuentes de calor en el caso de la necesidad de abrigo, los alimentos en el caso de la alimentación y el agua en todas sus presentaciones para el caso de la hidratación. Desde la teoría económica neoclásica, estos bienes se califican como escasos, siendo fácil la asignación de un precio por la interacción entre su oferta y demanda, lo que permite su adquisición en el mercado. Sin embargo, en esta clasificación es difícil agregar satisfactores como, tiempo bajo la protección de otro ser humano en el caso de la NMV de amparo, el tiempo en calidad y cantidad dedicado a dormir en el caso del descanso, y la cantidad de litros de aire consumido de la atmosfera para satisfacer la oxigenación. El servicio derivado de la contratación de mano de obra calificada y un lugar adecuado para dormir, son satisfactores en el caso del amparo y descanso respectivamente, pero estos difícilmente pueden ser adquiridos por personas en riesgo de muerte que se encuentren solos, como el bebé que teniendo todas las comodidades puede morir en soledad. Entonces, ellos si pueden encontrarse en el mercado y ser transados, pero necesitan de la intermediación de otro ser humano para ser consumidos (se ratifica la NMV de amparo); en lo que respecta al aire extraído de la atmosfera en el caso de la oxigenación podría calificarse según la teoría neoclásica, como un bien abundante ausente de precio porque la oferta es ilimitada y no existiría puja de la demanda para referir algún precio. Concepto bastante alejado de la realidad y per se, polémico.

En este sentido, se requiere una clasificación de los bienes y servicios destinados a la satisfacción de las $N M V$, para que la propuesta del IMV como método directo que relaciona la satisfacción de NMV con el consumo efectivamente realizado, evalúe mediante una encuesta sobre los bienes y servicios típicos y atípicos qué tienen a disposición y por tanto, si el grupo humano ha logrado satisfacerlas.

\section{El índice de mínimos vítales: Objetivo y formulación metodológica}

El objetivo del IMV es proveer un método directo de identificación de seres humanos en riesgo de muerte por insatisfacción de $N M V$, tomando en cuenta aspectos que no siempre se reflejan en el ingreso del hogar, parte de los datos cualitativos que ofrece la información arrojada por la encuesta como herramienta de estudio.

Para la construcción del índice utilizamos las siguientes variables:

1. Es: (escala de satisfacción) Variable que determina, según la encuesta, el nivel de satisfacción que tiene cada hogar para determinada NMV o determinado indicador interno a cada necesidad.

2. Nc: (nivel crítico de satisfacción) Nivel determinado de satisfacción de cada necesidad en el cual y por encima de él, se considera que la necesidad está satisfecha y por debajo se considera insatisfecha, nivel que establecimos en 0.5 
3. Is: indicador de satisfacción. Función matemática que determina mediante el manejo de las anteriores variables si existe presencia de insatisfacción de la NMV analizada, o del indicador interno a ella.

La modelación matemática se presentada a continuación:

$$
\text { Is: } E|(E s-N c)+1|
$$

En la función Is se utiliza la función matemática parte entera. El paréntesis (Es-Nc) expresa la distancia vectorial que existe entre el nivel crítico de satisfacción y la escala de satisfacción investigada en el hogar, será llamada Brecha de insatisfacción (Bi). Para obtener su magnitud es necesario someterla a la función valor absoluto y normalizar su dirección. Así:

$$
|B i|:|E s-N c|
$$

La Is se reformula:

$$
\text { Is: } E|B i+1|
$$

A continuación exponemos los valores que puede arrojar la función Is.

Tabla 1.Valores aleatorios de la función Is

\begin{tabular}{|c|c|c|c|}
\hline ss & Nc & $\boldsymbol{B i}$ & Is \\
\hline 0 & 0.5 & -0.5 & 0 \\
\hline 0.25 & 0.5 & -0.25 & 0 \\
\hline 0.5 & 0.5 & 0 & 1 \\
\hline 0.75 & 0.5 & 0.25 & 1 \\
\hline 1 & 0.5 & 0.5 & Fuente: Elaboración propia. \\
\hline
\end{tabular}

De lo anterior, sí el nivel crítico es $N c=0.5$, los niveles de satisfacción $E s=(0.5 ; 0.75 ; 1)$ al estar por encimas del Nc, indican que la NMV relacionada está satisfecha; para este caso, el resultado de la función Is será 1 (necesidad satisfecha para el hogar determinado). Y por otro lado, con los niveles de satisfacción $E s=(0 ; 0.25)$ evidentemente por debajo del Nc la NMV esta insatisfecha, en esta ocasión la Is tendrá como resultado 0 . Por tanto, 1 es satisfecho y 0 es insatisfecho.

Cabe aclarar, que será menester de la comunidad académica y de nuestro compromiso con Economía para no morir llevarlo a la práctica probando su efectividad.

\section{Alcance del IMV en las comunidades}

A diferencia de indicadores como el NBI que pretenden cuantificar el fenómeno de la pobreza, la especificidad del IMV se acerca a la insatisfacción de las necesidades más básicas o apremiantes en los hogares, individuos o comunidades, no a una solución totalitaria de pobreza. 
Esta propuesta busca atención y protección para los más necesitados, bajo el precepto que las desigualdades sociales son potenciales creadoras de desigualdades en términos de salud, "[...] una sociedad basada en el derecho a la vida de todos y todas, [...] implica necesariamente el reclamo de la vida de la naturaleza entera" (Hinkelammert y Mora, 2006, p. 348). Por tanto, el propósito de sus resultados es visibilizar una problemática social que pone en riesgo directo de muerte a seres humanos, y luego permitir a los economic policy makers desarrollar políticas públicas adecuadas para la protección de aquellas personas en situaciones de indignidad en el caso de la pobreza extrema o mínimamente disminuir la cantidad de personas que mueren directamente a causa de la insatisfacción de una NMV, "[...] Mientras que para el pensamiento neoclásico y neoliberal, la asociación y la solidaridad entre los seres humanos es vista como una distorsión, para una economía orientada hacia la vida son el medio para disolver estas "fuerzas compulsivas de los hechos"”' (Hinkelammert y Mora, 2006, p. 264) poniendo en escena los mínimos derechos de bienestar.

\section{Algunos efectos del trabajo en la satisfacción de las $N M V$ y su metabolismo con el planeta tierra}

La posibilidad de satisfacción de todas las necesidades humanas se da a partir del reconocimiento de la vida como condición, sujeto a la satisfacción de las NMV como derechos sociales de todos y todas. De ahí que el análisis de Economía para no morir, concluya en la interacción del ser humano con el planeta tierra, a saber, su metabolismo con la naturaleza; Para ello, los conceptos de actividad vital y cuerpo inorgánico propuestos por Carlos Marx (1844/1968) en los Manuscritos de Economía y Filosofía, permiten el vínculo de las NMV con las actividades humanas dispuestas a cada fin y la forma de apropiación de la naturaleza como su cuerpo inorgánico.

La actividad vital se define como la posibilidad que tiene todo ser vivo de satisfacer sus necesidades; para su realización, los seres humanos cuentan con una particularidad que los hace conscientes de elegir la forma en que quieren satisfacer sus necesidades, oportunidad que está ausente en las demás especies (para un animal carnívoro no existe otra posibilidad que cazar su presa mientras que el ser humano puede escoger entre cultivarlo, cazarlo o comprarlo). Es en este sentido que la intermediación entre el objeto de satisfacción y la forma en que se satisface la necesidad es reconocida como actividad vital humana, pues para comer, necesitamos sembrar, cazar o cosechar, de lo contrario la satisfacción de las necesidades no logra concretarse.

Aquel distanciamiento permite establecer una relación intermediada entre las necesidades y los satisfactores del ser humano, una forma de sobrevivencia propia de necesidadactividad vital -objeto de su necesidad, mientras que para los animales la relación es necesidad-objeto de su necesidad. Esas formas de satisfacer las necesidades humanas o de llevar a cabo su actividad vital han sido denominadas trabajo, definido como la posibilidad que tiene el ser humano de utilizar los recursos disponibles para garantizar su permanencia en la vida. El trabajo no solo como medio para producir, al representar la actividad vital, en sí mismo es un medio de transformación mediante el cual los seres humanos materializan sus necesidades con hechos, permitiendo el crecimiento personal; como ser social, se hace libre en tanto garantice su actividad vital. Por consiguiente, las NMV, a saber, abrigo, alimentación, amparo, descanso, hidratación y oxigenación, son necesidades siempre que se garantice la realización de las actividades de trabajo humano 
correspondientes a su satisfacción; cada una de ellas responde a una actividad vital característica:

\section{Ilustración 2. Actividades vitales relacionadas con las NMV}

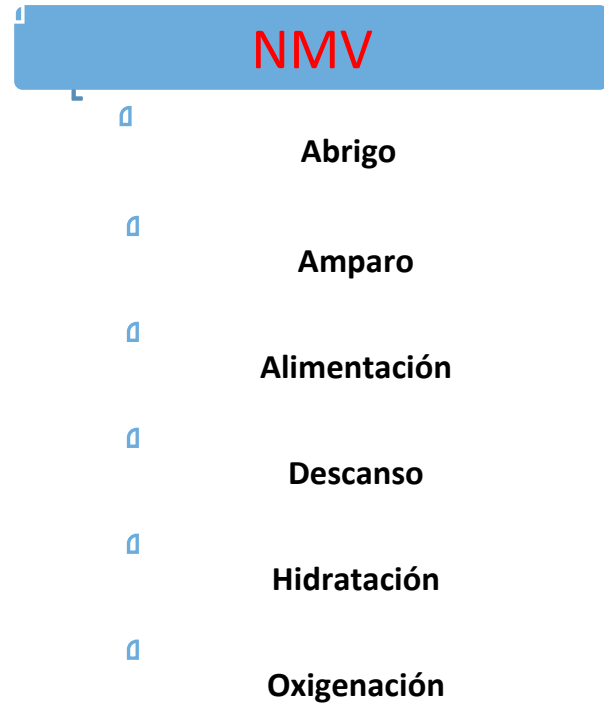

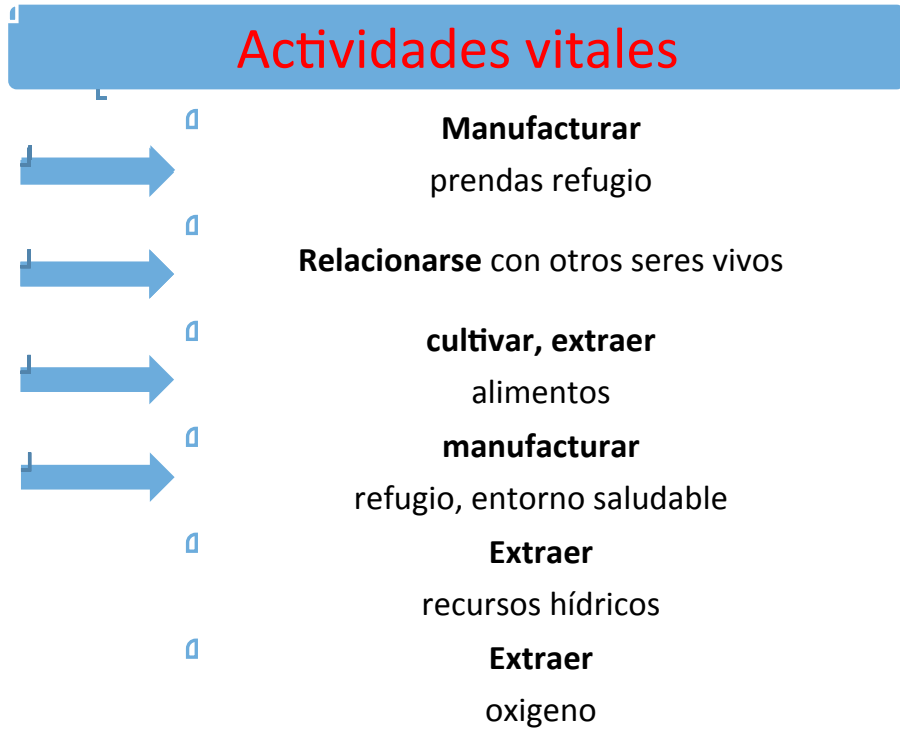

Bajo la racionalidad de la Actividad Vital, el ser humano hace de su entorno objeto de satisfacción, "[...] La naturaleza es el cuerpo inorgánico del hombre; la naturaleza, en cuanto ella misma, no es cuerpo humano. Que el hombre vive de la naturaleza quiere decir que la naturaleza es su cuerpo, con el cual ha de mantenerse en proceso continuo para no morir" (Marx, 1844/1968, p. 112). La naturaleza se constituye en cuerpo del ser humano pero al ser ajeno a su constitución fisionómica se denomina inorgánico, representando todas las extensiones de su cuerpo fisiológico logradas a través del trabajo. Solo así se entiende que la despensa no termina en la pared de la casa, ella se extiende a los cultivos de alimentos y estos cultivos vistos holísticamente no son más que una extensión inorgánica de nuestro estómago, toda vez que satisface de forma continua nuestra necesidad de alimentación, "[...] Sembramos la semilla, plantamos los árboles y fertilizamos el suelo. En fin, con nuestras manos y con variadas operaciones realizadas en este mundo, tratamos de fabricar, por así decirlo, otra Naturaleza" (Glacken 1956, p. 72). A continuación las manifestaciones del cuerpo inorgánico del ser humano en busca de la satisfacción de sus necesidades mínimas vitales, bajo la intermediación del trabajo como actividad vital: 


\section{Ilustración 3. Cuerpo Inorgánico en la satisfacción de las NMV}

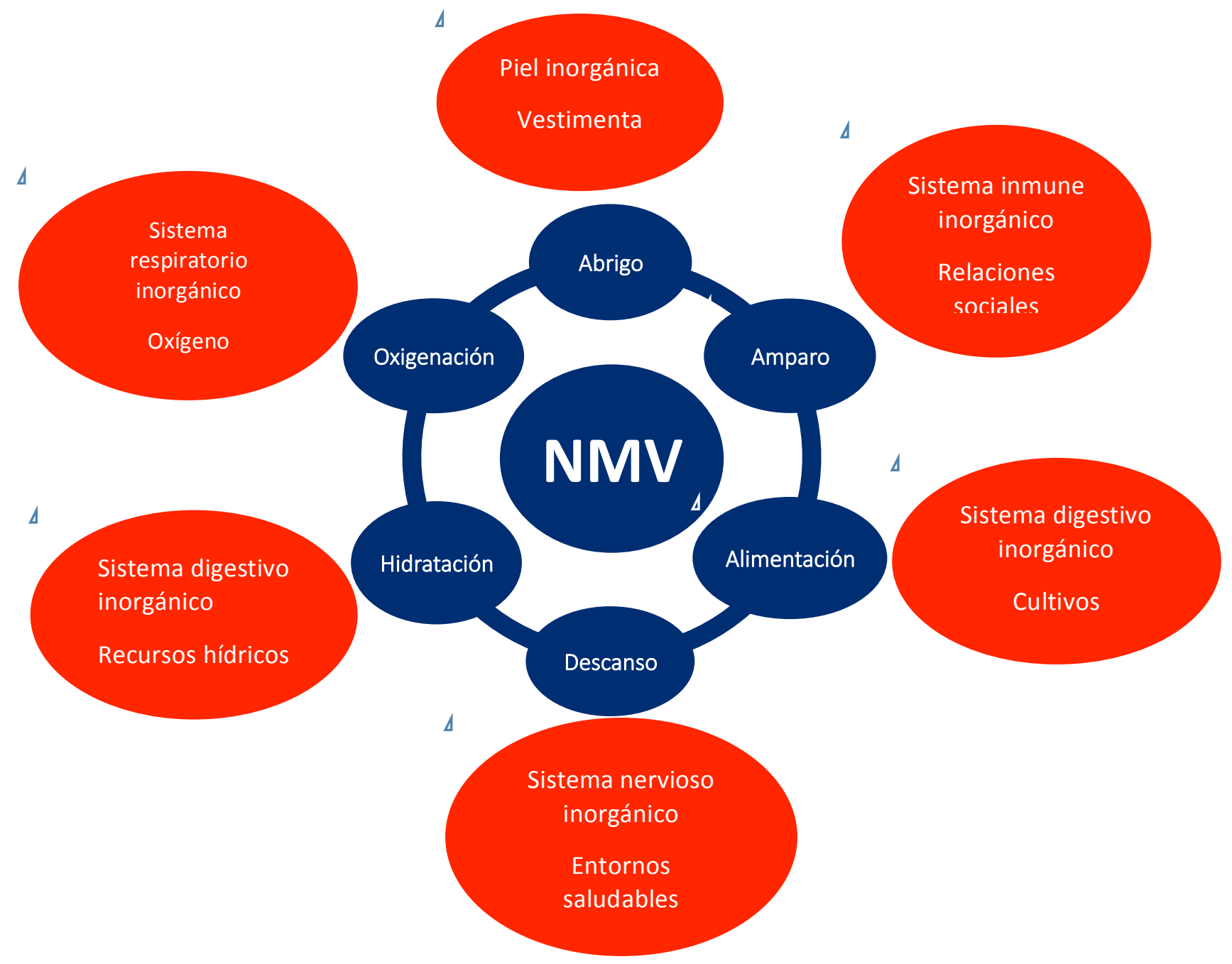

El ser humano posee diferentes extensiones de su cuerpo inorgánico, su comunidad, el territorio destinado a los cultivos, la naturaleza y los hábitats saludables se configuran en su piel inorgánica y sistemas inorgánicos: digestivo, respiratorio, inmune y nervioso. Todos dedicados a satisfacer las NMV mediante el trabajo involucrado a ellas.

Entendido el planeta bajo la cosmovisión del cuerpo humano dividido en orgánico, fisionomía de cada homo sapiens, e inorgánico, dedicado a satisfacer sus necesidades, 
ese nuevo cuerpo es sometido como organismo metabólico. $\mathrm{Y}$ aunque el término metabolismo tiene su origen en las ciencias naturales, de la misma forma en que una célula como unidad fundamental de la vida crea una membrana que la separa del entorno pero al mismo tiempo tiene una interrelación de otros compuestos celulares y de energía con él ambiente externo que la hacen crecer, metabolizarse y así reproducirse hasta dar paso a seres multicelulares, análogamente, existe un metabolismo concebido desde las $N M V$, a través del cual los seres humanos interactúan con la naturaleza y la transforman buscando su permanencia en su vida, ese metabolismo es la única fuente de suministro para que los cuerpos inorgánicos se materialicen.

\section{Ilustración 4. Metabolismo de las NMV con el planeta tierra}

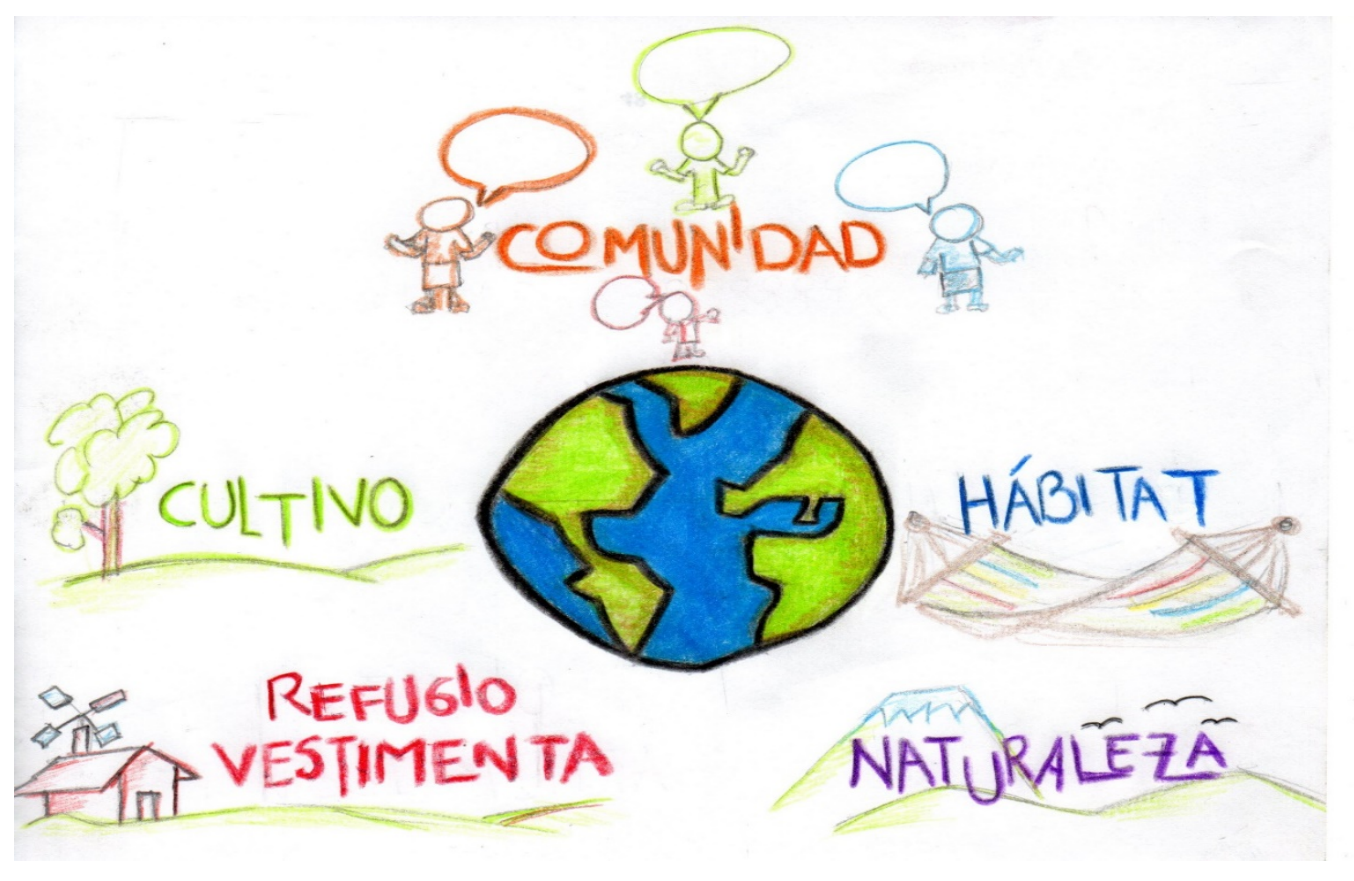

En un planeta finito como este, la apropiación de él como cuerpo inorgánico de una NMV desprovee a otra u otras $N M V$ del suyo, es decir, al ser el metabolismo del cuerpo inorgánico el conjunto de procesos de satisfacción de las necesidades humanas y en especial las $N M V$, si una de ellas es sobre-satisfecha, el planeta sufre un desequilibrio estructural y exponencial que se refleja en las desigualdades sociales, o en el peor de los casos, muerte de seres humanos. En estos terminos, no solo hablamos de unidad del ser humano con la naturaleza, sino de las formas en que el proceso historico de la actividad vital del trabajo ha modificado, roto y desequilibrado ese metabolismo con la naturaleza. La satisfacción del subconjunto de $N M V$ requiere la obtención de los satisfactores a esas necesidades que preservan la vida de los humanos, a saber, "existe relación causal entre la muerte de seres humanos por la insatisfacción de necesidades mínimas vitales y nuestra interacción metabólica con el planeta tierra mediante el trabajo como actividad vital"; y por tanto, dejando de lado toda institución humana que permita el intercambio de satisfactores de las necesidades entre humanos, la única fuente de satisfactores a esas $N M V$ es el planeta tierra y la forma de obtener esos satisfactores es mediante nuestra actividad vital, el trabajo. Esto reconfigura la propuesta colocando al planeta tierra en el 
centro del análisis de una Economía para no morir, que satisfaga antes que otra necesidad o deseo, las NMV. Una caracterización de la ilustración 4 muestra:

La comunidad: Cuerpo inorgánico que responde a la necesidad de amparo; es el sistema inmune inorgánico del ser humano en el proceso de satisfacción de esa NMV. En este sentido, la materialización del cuerpo inorgánico en comunidades es el resultado de la interacción metabólica que deviene de actividades vitales humanas sustentadas en el servicio, la protección y el amor hacia los que hacen parte de nuestra comunidad. Los seres humanos, agrupados en sociedad, no sólo comen, beben, crecen, y mueren, así como tampoco dedican toda la vida a construir estructuras, elaborar utensilios, instrumentos, armas o máquinas, aún más importante es el establecimiento de relaciones pues ésa parte intangible del ser humano opera como un armazón para los procesos de metabolismo, que crecen y enriquecen a través del tejido social garantizando el vivir al darle un sentido mismo al vivir.

Los hábitats: Forma de cuerpo inorgánico en el cual se satisface la necesidad de descanso; representa la posibilidad que tiene el ser humano de descansar en un lugar que le proporcione las condiciones adecuadas para dormir y evitar su deceso a causa de insomnio fatal. Tal hábitat funciona como el sistema nervioso inorgánico del ser humano, es decir, da las condiciones para que el ser humano pueda descansar. Dado que los hábitats humanos son diversos, se puntualiza el análisis en un hábitat personal compuesto por estructuras específicas para la satisfacción de las necesidades más personales, en este caso particular dormir. Elaboración de camas, hamacas, esteras, entre otras.

Refugio y vestido: Estas formas de cuerpo inorgánico del ser humano satisfacen la necesidad vital de abrigo, y por tanto son reconocidas como la piel inorgánica; representan la materialización de la única forma de estar protegidos contra altas o muy bajas temperaturas terrestres que lo afectan de manera directa y mortal. Pues a diferencia de los animales, los seres humanos no tienen plumas, escamas ni pelaje para proteger su cuerpo y por tanto para sobrevivir, requiere ropas y un refugio que lo proteja del rigor del clima.

La naturaleza: Responde a la configuración de cuerpo inorgánico que de forma análoga podría reconocerse como sistemas inorgánicos respiratorio y digestivo; por medio de ella se satisfacen las necesidades vitales de oxigenación y de hidratación. Tanto el aire como el agua son bienes que proporciona el planeta y por tanto deberían estar a disposición de todas y todos, sin embargo, en la medida que el ser humano ha desbordado procesos productivos que usan indiscriminadamente los recursos naturales, estas necesidades vitales han sido insatisfechas, diariamente mueren miles de personas alrededor del mundo por la carencia de ellas. Bajo su dinámica de adaptación ha modificado la naturaleza en un largo proceso evolutivo de desarrollo cultural, y por eso la naturaleza representa para él, la única forma de satisfacción de todas sus necesidades humanas.

Todas estas representaciones sin excepción, encarnan en su más amplia expresión al ser humano y su interacción metabólica con el planeta tierra. La manera en que se metabolizan las $N M V$ con el planeta muestra la forma primaria de intercambio entre la sociedad humana y la naturaleza, entendida a partir de la relación existente entre aquellas necesidades humanas, social e históricamente determinadas con los desequilibrios que se presentan. Si los seres humanos somos un fin y no un medio, una economía que grita 
"sálvese quien pueda o quien tenga como" distorsiona realidades, desvanece ideales y mata lentamente el espíritu humano, pero "¿En qué están empleando su tiempo las y los economistas, nuestros políticos, educadores sociales, medios de información?" (Maturana y Varela, 1996, p. IX), este fue el largo camino para explicar porque el uso extractivo indiscriminado del planeta y los recursos naturales de nuestro país ocasionaron la muerte de las y los guajiritos, a ellas y ellos todo este esfuerzo.

\section{Conclusiones}

La vida humana es condición irrefutable para la satisfacción de todas las necesidades y por tanto, la satisfacción de las NMV como subconjunto base de la necesidad de subsistencia es indispensable porque actúan como condición para la satisfacción de las demás necesidades humanas y no solo como satisfactores sociales. La insatisfacción de ellas, sea por extrema pobreza o por la carencia de condiciones mínimas de salud implica personas vulneradas en su condición de vida por tanto, es determinante el desarrollo de políticas públicas que reconozcan las $N M V$ como derechos sociales de todos y todas, y que direccionen esfuerzos en mayor bienestar para las comunidades.

Aclaramos que el IMV no es un indicador de pobreza sino de permanencia en la vida, es una herramienta metodológica que brinda la oportunidad de acercarse a la realidad social poniendo en contexto la situación de los y las ciudadanas colombianas, u otro lugar del mundo. La propuesta coloca en escena un mecanismo de acción que los economic policy makers podrán usar para el desarrollo adecuado de políticas públicas, con el objetivo de evitar las muertes de más personas a raíz de la insatisfacción de alguna NMV.

Equivocadamente, los seres humanos hemos concebido al planeta Tierra como depósito natural y por ello, históricamente ha respondido a extensiones inorgánicas del cuerpo humano que garantizan la satisfacción de las necesidades humanas. Sin embargo, la conservación del planeta es determinante para la permanencia de la vida y por ello se ubica en el centro del análisis, bajo la perspectiva que tal como otros animales, los seres humanos son una especie más que lo habita y por tanto es impropio concebirlo en su función. Es necesario asumir una nueva perspectiva de vida que permita entender la vida humana en función del planeta tierra y no al contrario.

Como la interacción metabólica ser humano - planeta tierra depende directamente de los procesos de actividad vital vinculados a la satisfacción de las NMV y demás necesidades humanas, existe una relación entre la muerte de seres humanos por insatisfacción de NMV y su interacción metabólica con el planeta tierra en el proceso de satisfacción de todas las necesidades humanas. El planeta representa la forma en la que se materializan tales necesidades y por tanto, es impropio sobre-satisfacer una necesidad a otra, ya que debido a su finitud la destinación de más recursos hacia un solo fin produce desequilibrios sociales, económicos y ambientales que lo afectan de manera directa y por la misma vía acaban con la vida humana.

Cerramos siendo reiterativos en que Economía para no morir es una apuesta teórica que pone la vida de los seres humanos por encima de todo derecho fundamental, y por tanto en su concepción exige que a todo ser humano le sea garantizada la vida por medio de la satisfacción de las $N M V$, este será el primer paso para garantizar un mayor bienestar a las comunidades, esta es nuestra apuesta por un mundo diferente. 


\section{Referencias}

Doyal, L. y Gough, I. (1994). Teoría de las necesidades humanas. Trad. Moyano, J. \& Colás, A. Barcelona: Icaria Editorial. Fuhem. (1991). A theory of Human Need. New York, USA: Palgrave Macmillan.

FAO, (2009). El estado de la inseguridad alimentaria en el mundo 2009, Crisis económicas: repercusiones y enseñanzas extraídas. Roma. P. 11. El derecho a la alimentación adecuada: Derechos Humanos. Folleto informativo N. 34. P.1

Glacken, C. (1967). Naturaleza y cultura en el pensamiento occidental desde la antigüedad hasta finales del siglo XVIII, Barcelona: Editorial del Serbal.

Hinkelammert, F. y Mora, J.H. (2008). Hacia una economía para la vida. Preludio a una reconstrucción de la economía. Editorial Tecnológica de Costa Rica, segunda edición, Cartago - Costa Rica.

Marx, K. (1968/1844). Manuscritos de economía y filosofía. Madrid: Alianza editorial.

Maturana, H y Varela, F (1996). El árbol del conocimiento. Las bases biológicas del conocimiento humano. Ed. Pensamiento y Debate, primera edición, Madrid - España.

Max Neef, M., Elizalde, A. y Hopenhayn, M. (1993) con la colaboración de Herrera, F., Zemelman, H., Jatobá, J. y Weinstein, L. Desarrollo a escala humana. Una opción para el futuro. Development Dialogue, Número especial. Santiago de Chile y Uppsala, Suecia: Cepaur y Fundación Dag Hammarskjöld.

Organización de las Naciones Unidas para la educación, la ciencia y la cultura (2005, octubre, 19). Declaración Universal sobre Bioética y Derechos Humanos de la UNESCO. Conferencia General. http://portal.unesco.org/es/ev.phpURL ID=31058\&URL_DO=DO_TOPIC\&URL_SECTION=201.html

Proyecto del Milenio de las Naciones Unidas, Halving Hunger: It Can Be Done (United Nations Publication, Sales No. 05.III.B.5), págs. 2 a 4. 